\section{A public policy up in smoke}

Canada has apparently decided to forego updating and enhancing the warning labels on cigarette packs in spite of evidence of their usefulness in the fight against smoking and of years of testing of new messages and images by Health Canada at substantial cost. The war on tobacco is by no means won. It remains one of the most important preventable causes of death, disability and lost quality of life in the country. Although smoking prevalence has declined over the past several decades, about one in five Canadians still smoke. Much progress can still be achieved to reach more addicted smokers who have found it difficult to quit and to prevent smoking uptake by youth.

Past successes in the fight against tobacco result from a comprehensive set of measures aimed, among other goals, at decreasing the social acceptability of smoking. The introduction of graphic images and enhanced warnings on cigarette packs has been an important component of Canadian tobacco control strategy and should be pursued with even more explicit messages and images. Although Canada pioneered the introduction of these labels over a decade ago, it is now rapidly falling behind other countries in this simple and effective smoking reduction strategy.

This change in policy is even contrary to the recommendations of the task forces created by the government to propose integrated and comprehensive strategies for cancer control and for cardiovascular disease control. Rather than acknowledging the benefits of strong and progressive tobacco control strategies, the government has stated that its new priority will be fighting the import of contraband cigarettes in the country. This new policy is more likely a smoke screen and its actions suggest that the true aim is to support the economic growth of the tobacco industry, even if it means undermining the health of Canadians.

While we should not expect all government decision making to be driven predominantly by public health consideration, there should be a minimum amount of common sense and decency when formulating policies which have important effects on the health of Canadians. Rather than trying to bolster death industries, such as big tobacco and the asbestos industry, the government should consider the impact of its policies on the health and wellbeing of its citizens, realizing that a healthy population is more productive and contributes to economic growth and social progress. We need more than ever to marginalize the tobacco industry, which has killed too many Canadians over too many decades.

Gilles Paradis

Scientific Editor

\section{Une politique publique qui part en fumée}

Le Canada a, semble-t-il, renoncé à actualiser et à améliorer les étiquettes de mise en garde sur les paquets de cigarettes, malgré les preuves de leur utilité dans la lutte antitabac et les années consacrées par Santé Canada à la mise à l'essai de nouveaux messages et d'images, à un coût considérable. La guerre contre le tabac est loin d'être gagnée. Le tabagisme est toujours l'une des plus importantes causes évitables de décès, d'invalidité et de perte de qualité de vie au pays. Bien que sa prévalence soit en baisse depuis plusieurs dizaines d'années, environ un Canadien sur cinq fume encore. Il reste beaucoup à faire pour joindre les fumeurs dépendants, ceux qui ont le plus de mal à renoncer à la cigarette, et pour empêcher que les jeunes ne commencent à fumer.

Les victoires passées contre le tabagisme ont été remportées grâce à un vaste ensemble de mesures qui visaient, entre autres, à réduire l'acceptation sociétale du tabagisme. L'ajout d'images crues et les avertissements plus sévères sur les paquets de cigarettes ont été un important élément de la stratégie antitabac canadienne; il faudrait poursuivre dans cette voie avec des messages et des images encore plus explicites. Le Canada a été le premier pays à apposer ces étiquettes il y a plus de 10 ans, mais d'autres en sont aujourd'hui rendus beaucoup plus loin dans l'emploi de cette stratégie simple et efficace pour réduire le tabagisme.

Ce changement d'orientation est contraire aux recommandations des groupes de travail créés par le gouvernement lui-même : ces groupes proposent des stratégies globales et intégrées pour lutter contre le cancer et les maladies cardiovasculaires. Plutôt que de reconnaître les avantages de stratégies antitabac musclées et progressives, le gouvernement a déclaré que sa priorité sera désormais de combattre l'importation des cigarettes de contrebande. Cette nouvelle politique est probablement un écran de fumée; on voit d'après ses interventions que son véritable objectif est de favoriser la croissance économique de l'industrie du tabac, même s'il faut pour cela miner la santé des Canadiens.

Nous ne pouvons pas nous attendre à ce que le gouvernement prenne ses décisions principalement pour des raisons de santé publique, mais il devrait faire preuve d'un minimum de sens commun et de décence lorsqu'il formule des politiques qui ont d'importants effets sur la santé de la population canadienne. Au lieu d'essayer de stimuler des industries de la mort, comme les grandes compagnies de tabac et l'industrie de l'amiante, il devrait examiner l'impact de ses politiques sur la santé et le bien-être de ses citoyens et se rendre compte qu'une population en bonne santé est plus productive et contribue à la croissance économique et au progrès social. Plus que jamais, nous devons marginaliser l'industrie du tabac, qui tue trop de Canadiens depuis trop longtemps.

Le rédacteur scientifique, Gilles Paradis 